\title{
Design of Microstrip Low-pass and Band-pass Filters using Artificial Neural Networks
}

\author{
HATHAT Ahmed \\ Faculty of sciences and Technology, University of Djelfa , Algeria \\ hathat.ahmed@gmail.com
}

\begin{abstract}
Usually the design of microstrip filters is done using simulators and classical approximation methods as Butterworth and Chebyshev, these techniques takes a lot of time to run for designing filters. In this paper we develop a faster artificial neural network model for designing a microstrip low-pass and band-pass filters, when the input are the dimensions of filter, operating frequency, the features of substrate, and the output are the transmission and reflection coefficients. The database uses for training this model is generated by a linear simulator based on circuits model. Two filters designed by the developed model are a stepped impedance low-pass filter with a cut-off frequency of $1 \mathrm{GHz}$ and a parallel coupled-line band-pass filter with fractional bandwidth of $25 \%$ and a central frequency of $2.45 \mathrm{GHz}$.

The proposed model has been shown to be as accurate as a linear simulator and much more efficient computationally in the design of microstrip low-pass and band-pass filters.
\end{abstract}

Keywords: artificial neural network model, microstrip low-pass and band-pass filters, parallel coupled-line, stepped impedance.

\section{INTRODUCTION}

Filters play an important role in many applications in radio frequency and microwave fields. They are used to select or confine signals within the assigned spectral limits. Microwave filters can be designed in various transmission line structures, such as waveguides, coaxial line and microstrip [1]. Microstrip line is one of the most popular types of planar transmission lines because it can be fabricated by photolithographic processes and is easily integrated with both passive and active microwave devices and offer a high range of frequency. The advantages of microstrip filters are their planar structure, low cost, insensitivity to manufacturing tolerances, reproducibility, a wide range of bandwidth and easy design process [2]. Development of simulation tools for microwave circuits remains a major challenge in microwave fields. There have been extraordinary recent advances in computer-aided design (CAD) of microwave circuits. They have been implemented in software tools and are being applied to microwave filters simulation, modeling and design using CAD, the costs for design and tuning can be reduced greatly. CAD can provide more accurate design, reduces the labor intensiveness and decreases the time from design to production. Furthermore, if the materials used are expensive, the first-pass design or less iteration afforded by CAD will reduce the extra cost of materials and other factors necessary for developing a satisfactory prototype $[1,3]$.

Using artificial intelligence techniques in the field of microwaves has produced powerful results [4-8]. Artificial neural network is one of these techniques, it can be used for modeling nonlinear multidimensional relationships. The evaluation time of a neural network model is also fast. For these reasons, neural networks have been used for various modeling and design applications including passive microwave circuits, in reference [5] the authors designed a low-pass filter by neural network in cut-off frequency of $0.5 \mathrm{GHz}$ and $0.7 \mathrm{GHz}$, in reference [6] the authors designing microstrip high-pass filter by neural network and focused their work in cut-off frequency of $1.5 \mathrm{GHz}$ and in reference [7] the designing of stepped impedance microstrip low-pass filters using artificial neural network at $1.8 \mathrm{GHz}$ is achieved by the authors. In [9] the design and analysis of stub microstrip band-pass filter at mid-band frequency 1.8 $\mathrm{GHz}$ is down using ANN and in [10] a presented design approach for a $N / 2$ resonator bandpass filter by using the artificial neural network modeling technique when the input variables are the dimensions of filter.

In this paper we present our contribution to the simulation of low-pass and band-pass microstrip filters using artificial neural networks for all operating frequencies, in 
order to develop a precise neural network model for simulation of microstrip filters. We have generated a database using a program that simulates the microstrip filter where the inputs are the filter dimensions, all range of operating frequency, the height of substrate and the relative dielectric constant, the outputs are the transmission (S21) and the reflection (S11) coefficients. This database is to train our neural network model such as the input variables are filter dimensions and the output is frequency response. This paper is organized into three sections: section 1 presents the design process of the low-pass and band-pass microstrip filters, section 2 focuses on modeling the filter and formulation of the problem and section 3 is dedicated to the presentation of simulation results.

\section{DESIGN PROCESS OF FILTERS}

The design of microstrip filters involves two main steps. The first is to select an appropriate low-pass prototype (in case of other filters: band-pass, high-pass, ,...etc, the transformation to a low-pass is done firstly), the choice of the type of response (Butterworth, Chebyshev ..), including the pass-band ripple and the number of reactive elements, will depend on the required specifications. The element values of the lowpass prototype filter, which are usually normalized to make a source impedance $g_{0}=1$ and a cut-off frequency $f_{c}=1$, are then transformed to the $\mathrm{L}-\mathrm{C}$ elements for the desired cut-off frequency and source impedance, which is normally $50 \Omega$ for microstrip filters. Having obtained a suitable lumped-element filter design, the next main step in the design of microstrip filters is to find an appropriate microstrip realization that approximates the lumped-element filter. A relatively easy way to implement low-pass filters in microstrip line is using alternating sections of very high and very low characteristic impedance lines. Such filters are usually referred to as steppedimpedance, or hi-Z, low-Z filters, and are popular because they are easier to design and take up less space than a similar lowpass filter using other technology [2]. For the band-pass filters, the parallel coupled line is one of the popular techniques used in the design of band-pass filters, it use half or quarter wavelength line resonators, the adjacent resonators are parallel to each other along half of their length [1] . In our study two examples of filters are chosen:

\section{A. Example 1 : Low-pass filter}

Cut-off frequency $\mathrm{f}_{\mathrm{c}}=1 \mathrm{GHz}$.

Maximal attenuation in bandwidth Amax = $0.1 \mathrm{~dB}$.

Load impedance equal source impedance $\left(\mathrm{Z}_{0}=\mathrm{Z}_{\mathrm{L}}=50 \mathrm{Ohm}\right)$.

\section{B. Example 2 : Band-pass filter}

Fractional bandwidth $\mathrm{FBW}=10 \%$

Center frequency $\mathrm{f}_{0}=2.45 \mathrm{GHz}$

Maximal attenuation in bandwidth Amax = $0.5 \mathrm{~dB}$.

Load impedance equal source impedance $\left(\mathrm{Z}_{0}=\mathrm{Z}_{\mathrm{L}}=50 \mathrm{Ohm}\right)$.

For example 1, we used the Butterworth approximation for the synthesis of the parameters of the desired filter. We find the order of the filter equal to 3 [2].

Figure 1 shows the low-pass prototype filter with normalized element values.

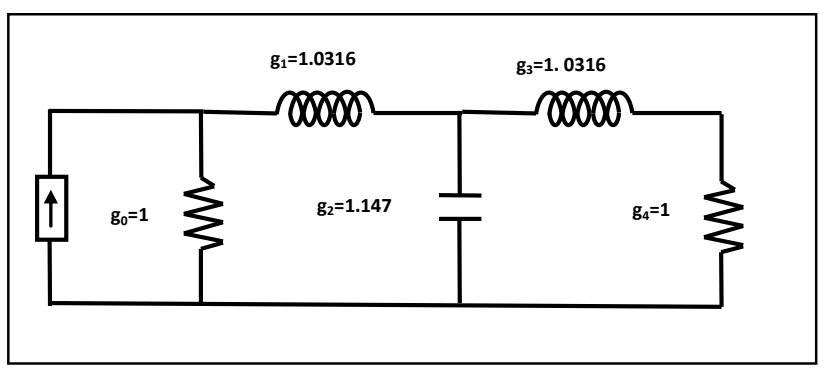

Fig. 1. Low-pass prototype filter.

The design of this filter on a relative dielectric constant substrate $\varepsilon_{\mathrm{r}}=10.8$ and a height $\mathrm{h}=$ 1.27 gives us the following dimensions: $W_{1}=$ $0.2 \mathrm{~mm}, \mathrm{~W}_{\mathrm{c}}=4 \mathrm{~mm}, \mathrm{~L}_{1}=9.81 \mathrm{~mm}$ and $\mathrm{L}_{\mathrm{c}}=7.11 \mathrm{~mm}$.

Figure 2 shows the layout of steppedimpedance microstrip low-pass filter.

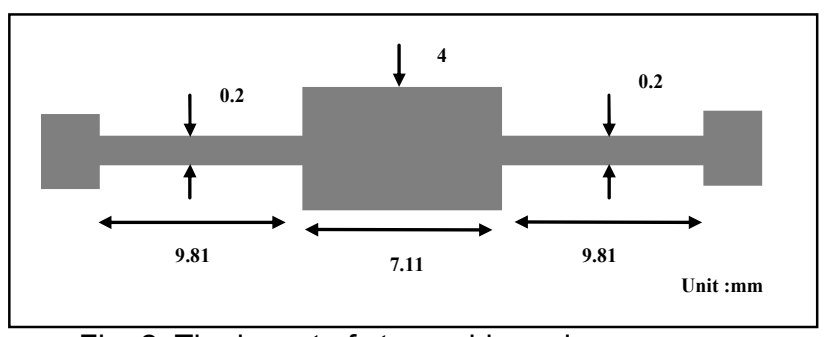

Fig. 2. The layout of stepped-impedance microstrip filter.

For example 2, we used the Chebyshev approximation for the synthesis the parameters of the desired filter. We find the order of the filter equal to 3 [2].

Figure 5 shows the low-pass prototype filter with normalized element values. 


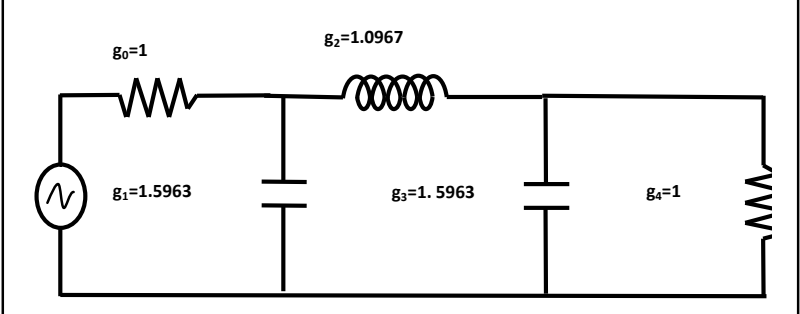

Fig. 3. Low-pass prototype filter

The design of this filter on a relative dielectric constant substrate $\varepsilon_{\mathrm{r}}=4.2$ and a height $h=$ $1.58 \mathrm{~mm}$ gives us the following dimensions: $\mathrm{W}_{1}=\mathrm{W}_{4}=2.54 \mathrm{~mm}, \mathrm{~W}_{2}=\mathrm{W}_{3}=3.53 \mathrm{~mm}, \mathrm{~S}_{1}$ $=\mathrm{S}_{4}=0.046 \mathrm{~mm}, \mathrm{~S}_{2}=\mathrm{S}_{3}=0.431 \mathrm{~mm}, l_{1}=$ $I_{4}=17.25 \mathrm{~mm}$ and $I_{2}=I_{3}=17.02 \mathrm{~mm}$.

Figure 4 shows the layout of parallel coupled line band-pass filter.

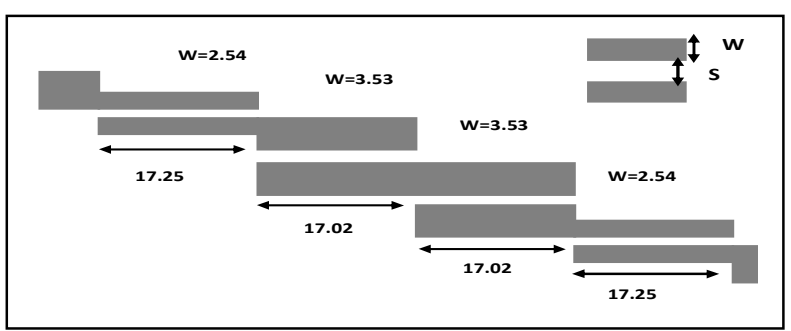

Fig. 4. The layout of parallel coupled line bandpass filter.

After the design of the filters, the simulation is done using an electromagnetic simulator such as IE3D, CST,.., etc $[11,12]$.

\section{PROBLEM FORMULATION}

Most filters are comprised of linear elements or components, linear simulations based on the network or circuit analyses are simple and fast for computer-aided analysis. Linear simulations analyze frequency responses of microwave filters based on their analytical circuit models. The filter shown in figure 2 consists of three section transmission line, and the scattering matrix $(A B C D)$ of a section transmission line is given by [1]:

$$
\left[\begin{array}{ll}
A & B \\
C & D
\end{array}\right]=\left[\begin{array}{cc}
\cos \theta & j Z_{c} \sin \theta \\
j \sin \theta / Z_{c} & \cos \theta
\end{array}\right]
$$

Such as $\theta$ is the electrical length and $Z_{c}$ is the characteristic impedance of the transmission line.

The filter shown in figure 4 consists of three parallel-coupled line, and the scattering matrix $(A B C D)$ of these lines is given by [1]:

$$
\begin{aligned}
& A=D=\frac{Z_{0 e} \cot \theta_{e}+Z_{0 o} \cot \theta_{o}}{Z_{0 e} \csc \theta_{e}-Z_{0 o} \csc \theta_{o}} \\
& B=\frac{j}{2} \frac{Z_{0 e}^{2}+Z_{0 o}^{2}-2 Z_{0 e} Z_{0 o}\left(\cot \theta_{e} \cot \theta_{o}+\csc \theta_{e} \csc \theta_{o}\right)}{Z_{0 e} \csc \theta_{e}-Z_{0 o} \csc \theta_{o}}
\end{aligned}
$$

$C=\frac{2 j}{Z_{0 e} \csc \theta_{e}-Z_{0 o} \csc \theta_{o}}$

Such as $\theta_{\mathrm{e}}$ and $\theta_{\mathrm{o}}$ are the electrical length for even and odd mode, $Z_{0 \mathrm{e}}$ and $Z_{00}$ are the characteristic impedance of even mode and odd mode of transmission line. The matrix $A B C D$ of these filters is calculated as follows:

$$
\left[\begin{array}{ll}
A & B \\
C & D
\end{array}\right]=\prod_{i=1}\left[\begin{array}{ll}
A & B \\
C & D
\end{array}\right]_{i}
$$

And the transmission $\left(\mathrm{S}_{21}\right)$ and reflection $\left(\mathrm{S}_{11}\right)$ coefficients are given by:

$S_{21}=\frac{2}{A+B / Z_{0}+C Z_{0}+D}$

$S_{11}=\frac{A+B / Z_{0}-C Z_{0}-D}{A+B / Z_{0}+C Z_{0}+D}$

The synthesis of these filters can be considered as a function of $Z_{1}, Z_{c}, \theta_{1}$ and $\theta_{c}$ for example 1 and $Z_{0 e}, Z_{0 o}, \theta_{0 e}$ and $\theta_{0 o}$ for example 2 . Using the equations they calculate the width and length of the microstrip line and the width, length and spacing of parallel-coupled line from the characteristic impedance and electrical lengths [2], we can calculate the transmission and reflection coefficients using the filter dimensions, substrate characteristics and operating frequency.

The first step is to generate a database to train the neural network model, where the inputs are the filter dimensions, the relative dielectric constant and the operating frequency, the outputs are the transmission and reflection coefficients. The next step is to validate the neural network model by a simulation of filters. 
Tables 1 and 2 shows the search space of the filter of low-pass and band-pass filters to generate the database.

TABLE 1. SEARCH SPACE OF LOW-PASS FILTER

\begin{tabular}{|c|c|c|}
\hline Dimensions & Min(mm) & Max(mm) \\
\hline $\mathrm{W}_{1}$ & 0.1 & 0.3 \\
\hline $\mathrm{L}_{1}$ & 6 & 10 \\
\hline $\mathrm{W}_{\mathrm{c}}$ & 2 & 6 \\
\hline $\mathrm{Lc}$ & 5 & 8 \\
\hline
\end{tabular}

TABLE 2. SEARCH SPACE OF BAND-PASS FILTER

\begin{tabular}{|c|c|c|}
\hline Dimensions & Min(mm) & Max(mm) \\
\hline $\mathrm{W}_{1}$ & 2 & 3 \\
\hline $\mathrm{L}_{1}$ & 0.04 & 0.05 \\
\hline $\mathrm{S} 1$ & 16 & 18 \\
\hline $\mathrm{W}_{2}$ & 3 & 4 \\
\hline $\mathrm{L}_{2}$ & 0.4 & 0.5 \\
\hline $\mathrm{S}_{2}$ & 16 & 18 \\
\hline
\end{tabular}

The architects of our neural network model for the simulation of low-pass and band-pass filter as shown in figure 5 and figure 6.

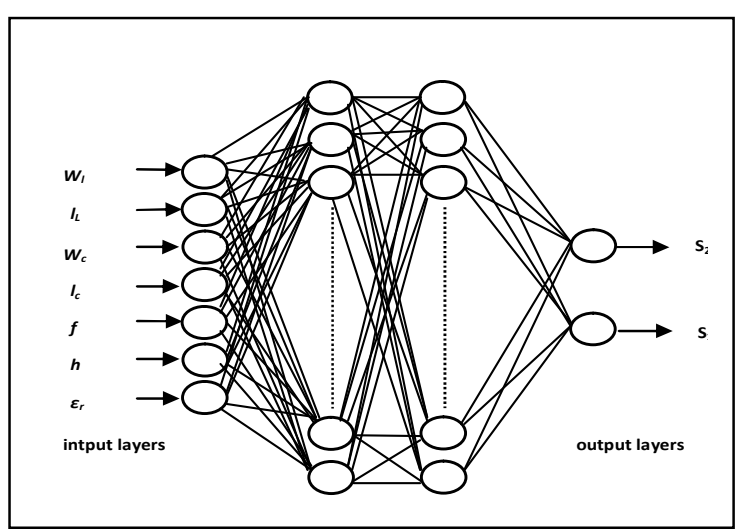

Fig. 5. Neural Network Model for designing a lowpass filter

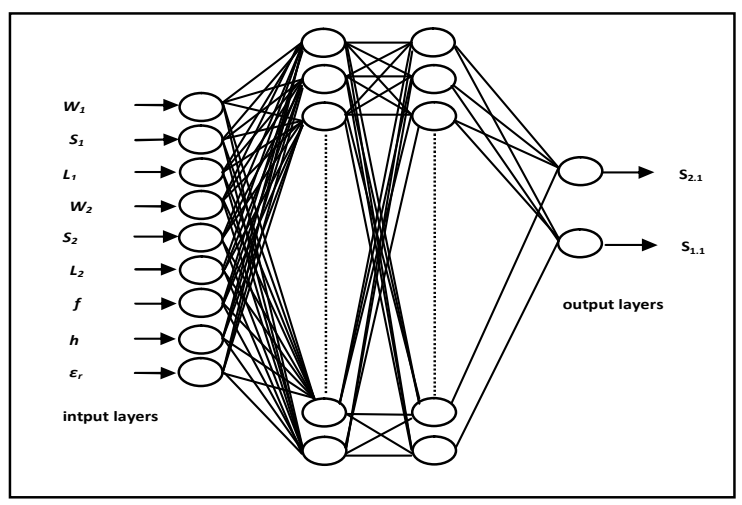

Fig. 6. Neural Network Model for designing a band-pass filter

Our database contains 400 filters for each application to train the neural network model.
There are four layers in our models: the input layer (contains 36 neurons for example 1 and 48 neurons for example 2), two hidden layers with 30 neurons for each layer and the output layer $\left(S_{21}\right.$ and $\left.S_{11}\right)$ with 60 neurons for example1 and 80 neurons for example 2. In our neural network model, the learning algorithm of Levenberg-Marquardt has been chosen, this algorithm is very efficient for the search for a minimum on the error function [5].

\section{RESULTS AND DISCUSSION}

Our contribution in this paper is the design of two microstrip filters by a linear simulator and the proposed artificial neural network model, a low-pass filter with a cutoff frequency of $1 \mathrm{Ghz}$ and a band-pass filter with a centre frequency of $2.45 \mathrm{Ghz}$ and fractional bandwidth of $10 \%$, these filters shown in figure 7 and figure 8 .

After training the neural network model by the database, these filters are simulated using the formulas (6) and (7), and then simulated by the proposed model .

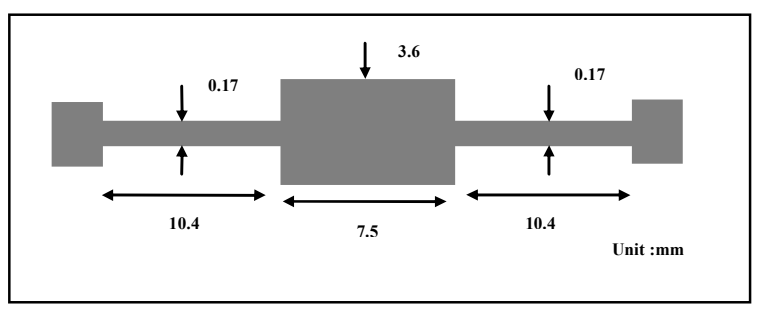

Fig. 7. Stepped-impedance microstrip low-pass filter.

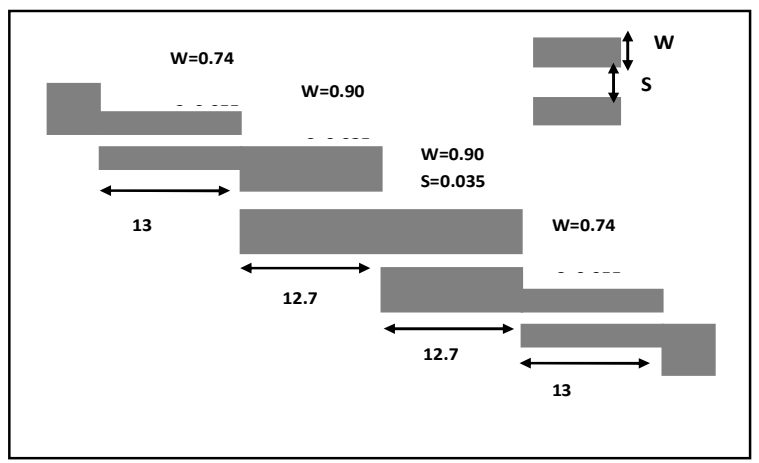

Fig. 8. Parallel coupled line band-pass filter.

Table 3 and table 4 shows the values of transmission $\left(\mathrm{S}_{21}\right)$ and reflection $\left(\mathrm{S}_{11}\right)$ coefficients of two filters obtained by linear simulator and obtained by the neural network model. 
TABLE 3. THE COEFFICIENTS S21 AND S11 VALUES

\begin{tabular}{|c|c|c|c|c|}
\hline $\begin{array}{c}\text { Frequency } \\
(\mathrm{GHz})\end{array}$ & $\begin{array}{c}\mathrm{S}_{21}(\mathrm{~dB}) \\
\text { simulator }\end{array}$ & $\begin{array}{c}\mathrm{S}_{11}(\mathrm{~dB}) \\
\text { Simulatol }\end{array}$ & $\begin{array}{c}\mathrm{S}_{21}(\mathrm{~dB}) \\
\mathrm{NNM}\end{array}$ & $\begin{array}{c}S_{21}(d B) \\
N N M\end{array}$ \\
\hline 0.1 & $-0,0083$ & $-30,1312$ & -0.0081 & -30.1511 \\
\hline 0.2 & -0.0293 & -23.6634 & -0.0281 & -23.6680 \\
\hline 0.3 & -0.0518 & -20.4059 & -0.0516 & -20.4172 \\
\hline 0.4 & -0.0610 & -18.7119 & -0.0601 & -18.7106 \\
\hline 0.5 & -0.0429 & -18.1608 & -0.0429 & -18.1705 \\
\hline 0.6 & 0.0109 & -18.7577 & 0.0116 & -18.7708 \\
\hline 0.7 & 0.0978 & -20.9592 & 0.0980 & -20.9278 \\
\hline 0.8 & 0.1986 & -26.5304 & 0.1975 & -26.5085 \\
\hline 0.9 & 0.2715 & -33.8822 & 0.2706 & -34.0362 \\
\hline 1.0 & 0.2514 & -20.5234 & 0.2519 & -20.5039 \\
\hline 1.1 & 0.0584 & -13.6657 & 0.0598 & -13.6615 \\
\hline 1.2 & -0.3762 & -9.3760 & -0.3754 & -9.3747 \\
\hline 1.3 & -1.0769 & -6.4897 & -1.0754 & -6.4862 \\
\hline 1.4 & -2.0094 & -4.5304 & -2.0087 & -4.5323 \\
\hline 1.5 & -3.0981 & -3.2090 & -3.0993 & -3.2084 \\
\hline 1.6 & -4.2586 & -2.3212 & -4.2587 & -2.3220 \\
\hline 1.7 & -5.4207 & -1.7220 & -5.4219 & -1.7203 \\
\hline 1.8 & -6.5361 & -1.3134 & -6.5371 & -1.3138 \\
\hline 1.9 & -7.5750 & -1.0305 & -7.5782 & -1.0313 \\
\hline 2.0 & -8.5210 & -0.8316 & -8.5222 & -0.8303 \\
\hline 2.1 & -9.3663 & -0.6899 & -9.3636 & -0.6882 \\
\hline 2.2 & -10.1080 & -0.5877 & -10.1086 & -0.5885 \\
\hline 2.3 & -10.7459 & -0.537 & -10.7505 & -0.5128 \\
\hline 2.4 & -11.2812 & -0.4603 & -11.2743 & -0.4616 \\
\hline 2.5 & -11.7158 & -0.4225 & -11.7159 & -0.4239 \\
\hline 2.6 & -12.0518 & -0.3970 & -12.0531 & -0.3965 \\
\hline 2.7 & -12.2912 & -0.3815 & -12.2911 & -0.3815 \\
\hline 2.8 & -12.4355 & -0.3748 & -12.4361 & -0.3742 \\
\hline 2.9 & -12.4865 & -0.3763 & -12.4824 & -0.3761 \\
\hline 3.0 & -12.4453 & -0.3858 & -12.4429 & -0.3840 \\
\hline
\end{tabular}

TABLE 4. the coefficients s21 and s11 values

\begin{tabular}{|c|c|c|c|c|}
\hline Frequen & $\mathbf{S}_{\mathbf{2 1}}(\mathbf{d B})$ & $\begin{array}{c}\mathbf{S}_{\mathbf{1 1}}(\mathbf{d B}) \\
\mathbf{s i m u l a t 0} \\
\text { cy (GHz) }\end{array}$ & $\begin{array}{c}\mathbf{S}_{\mathbf{2 1}}(\mathbf{d B}) \\
\mathbf{N N m}\end{array}$ & $\begin{array}{c}\mathbf{S}_{\mathbf{2 1}}(\mathbf{d B}) \\
\mathbf{N N M}\end{array}$ \\
\hline 2.0 & -14.5967 & -0.0026 & -14.5938 & -0.0026 \\
\hline 2.1 & -13.6051 & -0.0041 & -13.6093 & -0.0041 \\
\hline 2.2 & -12.5649 & -0.0067 & -12.5677 & -0.0066 \\
\hline 2.3 & -11.4630 & -0.0111 & -11.4621 & -0.0110 \\
\hline 2.4 & -10.2826 & -0.0192 & -10.2820 & -0.0192 \\
\hline 2.5 & -9.0170 & -0.0344 & -9.0171 & -0.0345 \\
\hline 2.6 & -7.6548 & -0.0649 & -7.6544 & -0.0650 \\
\hline 2.7 & -6.1852 & -0.1296 & -6.1856 & -0.1296 \\
\hline 2.8 & -4.6130 & -0.2764 & -4.6127 & -0.2765 \\
\hline 2.9 & -2.9852 & -0.6332 & -2.9855 & -0.6331 \\
\hline 3.0 & -1.4795 & -1.5311 & -1.4797 & -1.5308 \\
\hline 3.1 & -0.4345 & -3.7075 & -0.4345 & -3.7078 \\
\hline 3.2 & -0.0314 & -9.2114 & -0.0315 & -9.2126 \\
\hline 3.3 & -0.0218 & -10.0135 & -0.0216 & -10.0119 \\
\hline
\end{tabular}

\begin{tabular}{|c|c|c|c|c|}
\hline 3.4 & -0.1073 & -6.5884 & -0.1072 & -6.5886 \\
\hline 3.5 & -0.1637 & -5.6945 & -0.1637 & -5.6947 \\
\hline 3.6 & -0.1714 & -5.6003 & -0.1713 & -5.6006 \\
\hline 3.7 & -0.1430 & -5.9759 & -0.1431 & -5.9760 \\
\hline 3.8 & -0.0988 & -6.7629 & -0.0986 & -6.7628 \\
\hline 3.9 & -0.0537 & -8.0569 & -0.0538 & -8.0564 \\
\hline 4.0 & -0.0196 & -10.2448 & -0.0194 & -10.2436 \\
\hline 4.1 & -0.0017 & -15.4312 & -0.0016 & -15.4268 \\
\hline 4.2 & -0.0030 & -14.1603 & -0.0034 & -14.1626 \\
\hline 4.3 & -0.0235 & -9.8353 & -0.0238 & -9.8349 \\
\hline 4.4 & -0.0599 & -7.8275 & -0.0598 & -7.8287 \\
\hline 4.5 & -0.1055 & -6.6208 & -0.1054 & -6.6216 \\
\hline 4.6 & -0.1489 & -5.8958 & -0.1486 & -5.8964 \\
\hline 4.7 & -0.1728 & -5.5812 & -0.1728 & -5.5816 \\
\hline 4.8 & -0.1588 & -5.7607 & -0.1586 & -5.7608 \\
\hline 4.9 & -0.0953 & -6.8404 & -0.0951 & -6.8405 \\
\hline 5.0 & -0.0122 & -11.2795 & -0.0123 & -11.2801 \\
\hline 5.1 & -0.0568 & -7.9377 & -0.0571 & -7.9493 \\
\hline 5.2 & -0.5458 & -3.2654 & -0.5457 & -3.2658 \\
\hline 5.3 & -1.6794 & -1.3440 & -1.6791 & -1.3442 \\
\hline 5.4 & -3.2212 & -0.5588 & -3.2205 & -0.5590 \\
\hline 5.5 & -4.8466 & -0.2464 & -4.8473 & -0.2463 \\
\hline 5.6 & -6.4073 & -0.1167 & -6.4072 & -0.1166 \\
\hline 5.7 & -7.8595 & -0.0590 & -7.8603 & -0.0590 \\
\hline 5.8 & -9.2082 & -0.0315 & -9.2074 & -0.0316 \\
\hline 5.9 & -10.4576 & -0.0176 & -10.4593 & -0.0177 \\
\hline 6.0 & -11.6304 & -0.0103 & -11.6282 & -0.0104 \\
\hline
\end{tabular}

Based on the values shown in table 3 and table 4 , the differance between the results of linear simulator and obtained by the proposed model is very small in ordre of $0.0005 \mathrm{~dB}$ for all range of operating frequency.

Figure 9 and figure 10 shows the magnitude of transmission and reflection coefficients obtained by the simulator and neural network for low-pass and band-pass filters.

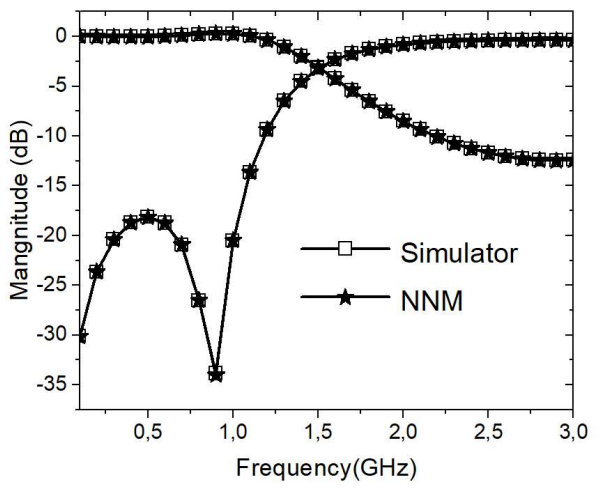

Fig. 9. The transmission and reflection coefficients of low-pass filter 


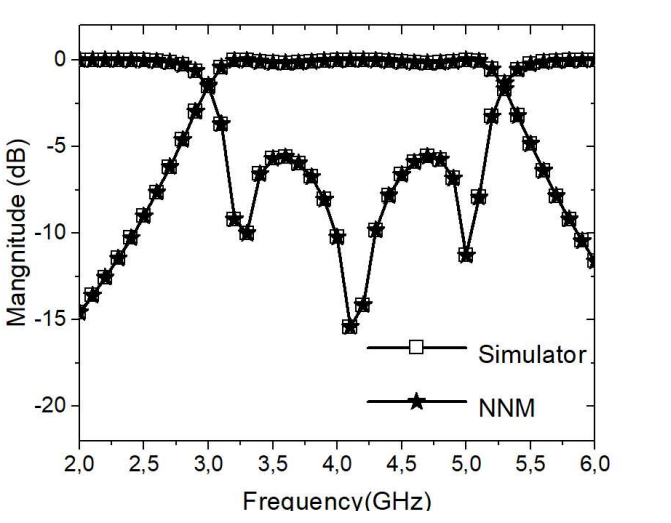

Fig. 10. The transmission and reflection coefficients of band-pass filter.

In figure 9 and figure 10, the results of proposed model is similar to the linear simulator in pass-band and stop-band frequency.

The design of these filters by the linear simulator takes a 5 minutes in $\mathrm{PC}$ of $\mathrm{CPU}$ 2.70GHz, RAM 8 Go and using Matlab $\mathrm{R} 2015$, and the same design take 30 seconds when used the proposed model.

The developed model provide a better result in term of transmission and reflection coefficients for all points in operating frequency when multiple publication focused only in cutoff frequency.

\section{CONCLUSION}

In this work a developed neural network model for designing microstrip low-pass and band-pass filters is presented. The strong point of this proposed model that works in pass-band/stop-band frequency and provide an accurate results and easy to use for designing microstrip filters.

The future work is applied this model for different technologies and many device like antenna and coupler.

\section{References}

[1] J. S. Hong, Microstrip Filters for RF Microwave Applications, $2^{\text {nd }}$ ed., J o h n Wiley \& Sons, 2011, pp. 1-12.

[2] D. M. Pozar, Microwave Engineering, 4th ed., John Wiley \& Sons, 2012, pp. 147-422.

[3] D. G. Swanson and W. J. R. Hoefer , Microwave Circuit Modeling Using Electromagnetic Field Simulation, Artech House Publ., Norwood, MA, 2003 , pp. 9-10.
[4] J. Narayana and K. Krishna, "High Dimensional Modeling of Microstrip Hairpin Band-pass Filter Using Artificial Neural Networks," International Journal of Future Generation Communication and Networking, vol. 5, no. 1, March 2012.

[5] G. S. Tomar, V. S. Kushwah and S. Saxena, "Design of Microstrip Filters Using Neural Network," IEEE The Second International Conference on Communication Software and Networks, Singapore,2010.

[6] V. S. Kushwah, F. M. Sharma and G. S. Tomar, "Analysis and Design of Microstrip High Pass Filters Using Artificial Neural Network," International Journal of Computer Information Systems and Industrial Management Applications, vol. 3, 2011, pp. 347-354

[7] V. S. Kushwah, G. S. Tomar and S. S Bhadauria, "Designing Stepped Impedance Microstrip Low-Pass Filters Using Artificial Neural Network at $1.8 \mathrm{GHz}$," IEEE International Conference on Communication Systems and Network Technologies 2013.

[8] Q. J. Zhang, K. C. Gupta and V. K Devabhaktuni, "Artificial neural networks for RF and microwave design: From theory to practice," IEEE Trans. Microwave Theory Tech, vol. 51, no. 4, pp. 1339-1350, 2003.

[9] G. S. Tomar, V. S. Kushwah and S. S. Bhadauria "Artificial neural network design of stub microstrip band-pass filters" Int. J. Ultra Wideband Communications and Systems, Vol. 3, No. 1, pp. 38-49,2014

[10] X. Li, J. Gao, J. G. Yook and X. Chen, "Bandpass Filter Design by Artificial Neural Network Modeling "Asia-Pacific Microwave Conference Proceedings Vol. 5, 2005.

[11] ANSYS, http://www.ansys.com/ products lacademic ,accessed 04 April 2021.

[12] Sonnet Software, Inc, http://www.sonnetsoftware.com/ products/sonnet-suites/ , accessed 04 April 2021. 